\title{
Design Distribuído: novas práticas e competências para o design pós-industrial
}

\author{
Distributed Design: new practices and competencies for the post-industrial design
}

\author{
COSTA, Christiane; Mestre; UFPR \& UTFPR \\ christiane.ogg@gmail.com \\ PELEGRINI, Alexandre; Doutor; UFPR \& UTFPR \\ avpelegrini@yahoo.com
}

\begin{abstract}
Resumo
As tecnologias de abordagem distribuída viabilizadas pela web 2.0 possibilitaram a abertura e compartilhamento de dados, a produção entre pares e novos comportamentos colaborativos, esmaecendo a linha que separava usuários, designers e produtores. As tecnologias de fabricação digital e o surgimento da quarta revolução industrial (Indústria 4.0) aceleram estas mudanças por meio da reconfiguração e relocalização da produção e do consumo, possibilitando novas formas de organização social, novos comportamentos produtivos, econômicos e ambientais. Neste contexto o foco do design se desloca do produto para uma abordagem complexa e sistêmica onde o designer tem um papel estratégico baseado nas interações sociais participativas. Mediante revisão bibliográfica, este artigo busca discutir a ampliação das possibilidades de atuação do designer e suas novas competências. A pesquisa aponta para a necessidade de expansão dos conceitos de design e a reavaliação das habilidades desenvolvidas atualmente para que o ensino superior de design possa responder a este novo contexto.
\end{abstract}

Palavras Chave: novas práticas; competências; ensino de design.

\section{Abstract}

Distributed approach technologies enabled by web 2.0 made possible the opening and sharing of data, peer production and new collaborative behaviors, blurring the line between users, designers and producers. Digital manufacturing technologies and the rise of the fourth industrial revolution (Industry 4.0) accelerates these changes through the reconfiguration and relocation of production and consumption, enabling new forms of social organization, new productive, economic and environmental behaviors. In this context, the focus of design shifts to a complex and systemic approach where the designer has a new strategic role based on the interaction between people, technologies, products and services. Through a bibliographical review, this article seeks to discuss the expansion of the possibilities of the designer and their new competencies. The research points to the need to expand the concepts of design and a reevaluation of the skills currently developed so that higher education in design can respond to this new context.

Keywords: new practices; Skills; design education. 


\section{Introdução}

O design de produto como profissão, antes do advento das tecnologias de abordagem distribuída e de produção digital, esteve vinculado ao sistema de produção industrial capitalista que prioriza a produção em massa para geração de valor econômico, restringindo a atividade de design a poucos modelos alternativos. Com a disseminação e o acesso as tecnologias web 2.0 e de produção digital, surge uma infinidade de possibilidades para geração de novos modelos de produção, novas práticas sociais, novos modelos de organização e negócio ampliando as possibilidades de atuação do design.

Para manter sua relevância o ensino de design precisa responder a este contexto proporcionando aos alunos uma compreensão destas mudanças de paradigma e não apenas acrescentando capacidades digitais a um portfólio de competências. $O$ ensino do design baseado na antecipação do futuro da sociedade deve prever estas novas possibilidades, revendo o perfil desejado para o designer, estabelecendo novas competências para que os futuros designers possam agir de maneira crítica e consciente sobre suas próprias decisões que potencialmente afetarão a qualidade de vida das pessoas.

São muitos os autores que apontam novas práticas para o design e questionam a situação atual do ensino do design pós-industrial (Findeli, 2001; Manzini, 2011; Poggenpohl, 2012; Wood, 2012; Celaschi e Formia, 2012; Wilson e Zamberlan, 2015; Imbesi 2011; Loy, 2015; De Vere, 2013; Troxler, 2015; Franzato, 2016). Por se tratar de um assunto comum entre muitos autores e uma preocupação emergente dos dirigentes e professores do ensino superior de design, mediante revisão bibliográfica, este artigo se propõe a discutir as possibilidades sobre o futuro do design para estabelecer um perfil preliminar deste profissional.

Conclui-se ao final do trabalho que as competências esperadas para os futuros designers colocam o ensino atual numa posição de reavaliação tanto de conteúdos quanto de estrutura pedagógica, levando em consideração o conhecimento complexo, necessário em várias esferas, que convencionalmente não fazem parte da prática disciplinar atual.

\section{Tecnologias de abordagem distribuída, fabricação digital e novas práticas para o design}

Os princípios da fabricação digital e de código aberto potencializam a democratização do design e da produção, configurando de formas diversas as práticas de design, produção, consumo e distribuição, misturando papéis, processos e funções. $\mathrm{O}$ acesso a comunidades globais combinadas com as tecnologias de baixo investimento inicial habilitam a cultura participativa criando novas oportunidades para estratégias de implementação de novos produtos.

Desta forma os designers podem se envolver diretamente com o usuário final para testar ideias e soluções de design, iniciar atividades de codesign ou design participativo, acessar grupos amplamente dispersos ou demograficamente segmentados, incentivando os participantes a se engajarem no resultado final (De Vere, 2013). O desenvolvimento de um novo design pode ser realizado por leigos, prosumers ${ }^{1}$, produsers ${ }^{2}$, comunidades criativas, experts de áreas diversas,

\footnotetext{
${ }^{1}$ Alvin Toffler cunhou o termo "prosumer" (produtor + consumidor) para descrever o surgimento de um consumidor de bens mais informado e envolvido num modelo de produção de demanda de itens customizados.

${ }^{2}$ Todos os participantes são usuários bem como produtores num papel hibrido onde o uso também é necessariamente produtivo. O produto deste será um artefato temporário de um processo contínuo de produção (Bruns, 2007).
} 
designers e empresas, ou mesmo pela interação entre estes grupos. Esta configuração potencializa a construção de redes de baixo para cima habilitando sistemas descentralizados, colaborativos, não proprietários, onde o designer deve ser capaz de colocar em prática a colaboração interativa.

Por outro lado, estas ferramentas virtuais de mídia social permitem a conexão entre pares, o compartilhamento, cocriação, remixagem e a modificação de conteúdo gerado, podendo ainda ser utilizadas para fornecer informações (data mining) para analisar o comportamento do consumidor, encontrar padrões ocultos e prever tendências sobre os possíveis usuários do sistema a ser projetado.

A interação entre as pessoas envolvidas no desenvolvimento destes projetos pode ocorrer através de encontros em espaços físicos, plataformas virtuais colaborativas, Fablabs, makerspaces, hackerspaces, ou de forma iterativa entre estes espaços. $\mathrm{O}$ acesso ao projeto ou produto realizado pode se dar por meio de abertura total (open design) ou abertura parcial baseada em licenças, ou apenas para download conforme o objetivo do projeto e a quem se quer beneficiar. Desta forma, um amplo mercado pode ser acessado sem a necessidade de instalações de produção e distribuição, à medida que os usuários fazem o download do design, customizam para suas exigências e produzem de forma independente o produto (De Vere, 2013).

A fabricação digital direta permite o desenvolvimento de design para produção de um único objeto sob demanda, onde o produto fabricado tem características únicas atendendo a necessidades específicas das pessoas. As tecnologias digitais integradas viabilizam também a fabricação distribuída de pequena escala possibilitando a produção de artefatos que utilizem informação em rede e recursos locais, potencializando o desenvolvendo local e possibilitando o abastecimento de recursos para locais de difícil acesso.

Outro mecanismo importante para viabilizar a produção de pequena escala é o financiamento coletivo (crowdfunding) que promove iniciativas criativas financiadas e realizadas por meio de apoio colaborativo. O lançamento de produtos, com baixo comprometimento financeiro ou risco inerente, viabiliza o teste de novas ideias quando estas ainda estão em fase de pré-produção, dando ao designer maior liberdade para experimentação, uma vez que o projeto não prossegue sem apoio financeiro suficiente.

As tecnologias de hardware de baixa complexidade tais como o Arduíno, têm aproximado os designers e profissionais de outras áreas, novos híbridos de design que misturam artes, engenharia, mecatrônica, moda, entre outros, estão surgindo incentivados pelas tecnologias digitais integradas, tornando a natureza emergente da prática de design cada vez mais difusa. Estas práticas incentivam a transdisciplinaridade, o aprendizado entre pares e o desenvolvimento de projetos complexos que só seriam possíveis em organizações de grande porte.

Estas mudanças no sistema de financiamento, produção e distribuição incentivam a autoprodução, onde o autor do projeto se torna responsável pela produção, comunicação e venda (Dias, 2014, Bianchini, 2014) - permitindo que um artesão, um designer ou qualquer pessoa capaz de projetar, empreenda por meio das tecnologias integradas disponíveis. Para o "Designer = empresa cunhado por Arquilla, Bianchini e Maffei (2011), "designpreneurs" por Borja de Mozota (2011) surge a possibilidade de autoprodução realizada fora da fabricação tradicional criando seu próprio nicho, produzindo e entregando bens para o mercado. Esta indústria emergente, situado entre o artesanal e a fabricação em massa tradicionais, forma um tipo de "Boutique Manufacturing", segundo Troxler (2015) que se torna um novo paradigma para o design. 
Num conceito mais amplo, o design rede ${ }^{3}$ ou "redes de projeto" tem como efeito mais significativo a organização e a contínua reorganização das relações e das atividades que são desenvolvidas no ecossistema das organizações.

A participação colaborativa de uma diversidade plural de atores, nas redes de design estratégico exige facilitadores de processos e mediadores de relações. As competências projetuais específicas dos designers podem ser utilizadas para que o diálogo e a construção coletiva de soluções sejam conduzidos. O design se transforma em uma plataforma transdisciplinar que sustenta a convergência dos especialistas e dos demais atores que integram essa produtiva rede de colaboração (Franzato, 2016).

A possibilidade de recapturar a tecnologia que foi retirada das mãos do artesão e agora potencialmente se transfere novamente às pessoas comuns faz com que possamos imaginar inúmeras possibilidades para transformar e reconfigurar novos contextos. A produção e o design distribuídos, desvinculados da indústria tradicional tem a oportunidade de considerar as tecnologias e atores totalmente livres da estrutura industrial tradicional vigente, criando um espaço de experimentação a fim de refletir sobre a complexidade do contexto de produção e consumo, propondo encontrar novas realidades sociais, novas formas de prática, novos modelos econômicos e novas formas de atribuir valor. Relaxar ou remover essas restrições permite ao designer imaginar possibilidades e alternativas e desafiar sistemas e papéis estabelecidos. Este enquadramento permite imaginar novos cenários onde pessoas e tecnologias digitais integradas configuram criativamente novas possibilidades de contextos socio-técnicos e socio-produtivos.

Nesta abordagem, a atribuição de valor também pode ser repensada de maneira transformativa depositando valor em produtos, sistemas e relações humanas que considerem objetivos sociais mais amplos. Estas práticas experimentais podem estar apoiadas em um dos elementos ou em múltiplos elementos combinados.

No outro extremo do espectro existe a emergência de uma nova revolução industrial que se propõe a maior digitalização, informatização, robotização, uso de dados e de redes de comunicação digital, capaz de atender o consumo personalizado de produtos de alta complexidade e variabilidade. A Alemanha, o Reino Unido, a Holanda, a China são alguns dos principais países que estão formulando políticas industriais para moldar ativamente o futuro da indústria 4.0. Segundo Hermann et al (2015) a indústria 4.0 promete eficiência operacional substancialmente aumentada e o desenvolvimento de modelos de negócio, serviço e produtos inteiramente novos causando um grande impacto econômico.

Sob esta perspectiva, o ensino do design necessita dirigir seus esforços para experimentar novas práticas capazes de desenvolver as potencialidades oferecidas pelas tecnologias, práticas sociais, modelos de produção, modelos de organização e negócio reconfigurando os modelos sócio técnicos e produtivos da era industrial (Menichinelli, 2016) e ampliando as atividades do designer e seu alcance social, econômico e sustentável.

Nestes sistemas emergentes as mudanças para a prática do design podem ocorrer: pela convergência entre tecnologias, novas práticas sociais, novos locais de produção e aprendizagem, novos modelos de negócio e cadeias de valor, novas formas de produção e consumo, entre outros (fig.1).

\footnotetext{
${ }^{3}$ Sistema aberto e dinâmico dos atores envolvidos em um projeto, articulados por meio de relações colaborativas.
} 


\section{Artigo Completo}

Figura 1 - novas oportunidades para prática do design

1. NOVAS TECNOLOGIAS E SUAS CONVERGÊNCIAS
-IMPRESSÃO 3D
-AUTOMAÇAO/ROBÓTICA
-INTERNE DAS COISAS
-INTELIGENCIA ARTIFICIAL
- REALIDADE VIRTUAL/AUMENTADA
- WEARABLES
- BIOTECNOLOGIA
-BIG DATA...

Fonte: a autora

Para Celaschi (2017) num futuro próximo a profissão do designer sofrerá mudanças consideráveis, pois o design vinculado à indústria 4.0 será realizado numa direção oposta da atual, começando pelo comportamento do consumidor, onde bens e serviços nascem e evoluem continuamente através do reprocessamento da informação que retorna do consumidor ou usuário. O comportamento do usuário será monitorado por meio do produto (internet das coisas) influenciando o indivíduo em todos os momentos. O desenvolvimento do produto e do modelo de negócios será simultâneo e integrado horizontal e verticalmente, onde todos os agentes trabalham em conjunto, dissolvendo as barreiras disciplinares atuais. Segundo Schuh et al (2016) o design para a indústria 4.0 requer uma nova abordagem. Devido aos ciclos de vida mais curtos do produto, a necessidade de produtos mais individualizados, a integração do consumidor no processo de design, a comunicação de dados do produto, a segurança cibernética e funcional, a criação de produtos inteligentes como suporte de informação, cresce a necessidade de uma abordagem holística sobre o "smart design" no contexto da Industria 4.0.

O relatório da OECD (2017) indica que essas mudanças provocadas pela Industria 4.0 afetariam pesquisa, atividades de desenvolvimento tecnológico e inovação, mercado de trabalho, distribuição de renda e bem-estar; habilidades; vários campos de regulação e ainda poderia ser um grande facilitador da economia circular.

A economia social baseada em redes e valores intangíveis reflete mudanças profundas que afetam as estruturas socioeconômicas modernas. O principal desafio do design é precisamente desenvolver e/ou suportar soluções altamente complexas que exigem uma visão ampla do projeto, envolvendo produtos, serviços e comunicação numa forma integrada e sustentável (Krucken, 2009).

Esta nova perspectiva para a prática do design requer um novo ensino, no entanto, em comparação com o desenvolvimento de práticas de design, o ensino tem se desenvolvido mais lentamente nas últimas décadas, e muitas escolas de design continuam a ensinar os seus alunos 
apenas as habilidades de projeto, métodos e processos (Kaufman, 1998).

\section{Novas práticas, novas competências e o ensino de design}

São muitos os autores que questionam a situação atual do ensino do design pós-industrial principalmente pela vinculação com os paradigmas da sociedade industrial que prioriza o ensino de habilidades técnicas em detrimento do potencial reflexivo e crítico (Whiteley, 1998; Buchanan, 2001), limitando a compreensão dos estudantes sobre seu papel como cocriadores do futuro e os distanciando da dinâmica de transformações sociais, culturais, econômicas e tecnológicas (Findeli, 2001; Manzini, 2011; Poggenpohl, 2012). Esta tendência mantém a maioria dos designers como contribuintes especializados e não como pensadores holísticos que trabalham para a sociedade como um todo (Wood, 2012).

Para Celaschi e Formia (2012), tanto o papel do design quanto o ensino do design ainda estão polarizados em dois modelos distintos: o modelo que absorve o design como uma metodologia fiel à concepção racional-funcionalista e outro mais disruptivo pensado na renovação do sistema de produção baseada numa redefinição dos papéis de produtor, consumidor e do designer. No modelo disruptivo os designers são vistos como agentes de mudança porque a importância estratégica do design está na sua atuação como identificador de problemas, nas decisões sobre o porquê, o que e como produzir.

A mudança de foco do design para a transformação de contextos mais amplos e questões sociais também expandiu a possibilidade de atuação do design. Este discurso ocorre há pelo menos três décadas com o objetivo de criar uma sociedade melhor (Bonsiepe, 2013), mas a possibilidade de interferência do design nas questões sociais, estando atrelado ao sistema industrial, era muita limitada. O novo discurso do design sobre transformação baseia-se na inovação com relevância social.

O design neste contexto passa a ter outras demandas onde os problemas não são bem definidos, as perguntas e respostas não são mais tão objetivas e o resultado passa a ser um enquadramento provisório, baseado no conhecimento possível naquele momento. As respostas extrapolam, muitas vezes, o âmbito do design: podem estar nas disciplinas antropológicas, sociológicas, psicológicas e de igual forma naquelas relacionadas à estética, neurociência, semiótica, sociologia, história, psicologia do conhecimento e da percepção, além de tantas outras das áreas humanistas e sociais que têm mais ou menos aproximação com o design (Celaschi e Moraes, 2013).

As competências profissionais e as divisões disciplinares também requerem novos modelos, pois não atendem à atual complexidade inerente às situações que emergem da prática. Segundo Loy (2015), o momento exige um repensar radical da pedagogia e do conteúdo, levando em consideração o conhecimento complexo necessário em várias esferas que convencionalmente não fazem parte da prática da disciplina do currículo tradicional.

Para alguns autores a atual complexidade oferecem novas oportunidades para práticas criativas hibridas que transpassam fronteiras conceituais e disciplinares e, a "indisciplinaridade" desponta como um modo particular de ação que deixa de ser disciplinar onde novas e inesperadas formas de trabalhar são criadas (Bremner e Rodgers, 2013, p. 12 appud Mineiro). Porém o conceito de transdisciplinaridade ligado tanto a uma nova visão como a uma experiência vivida, se adequa a necessidade do design em estabelecer a horizontalidade e a transversalidade de conhecimentos. Segundo Nicolescu (1997) a transdisciplinaridade é um caminho de autotransformação orientado 
para o conhecimento de si, para a unidade do conhecimento e para a criação de uma nova forma de ver e agir. Diz respeito ao que está, ao mesmo tempo, entre, através e além de todas as disciplinas. Seu objetivo é a compreensão do mundo presente, e um dos imperativos para isso é a unidade do conhecimento. $\mathrm{O}$ aperfeiçoamento ou desenvolvimento de competências transversais por meio da transdisciplinaridade contempla a criação de novas formas de ação.

Dubberly, (2011) afirma que a mudança no design não se relaciona apenas sobre o que é produzido ou como é produzido, mas é também uma mudança de visão de mundo, uma mudança de metáfora de enquadramento, uma mudança de estrutura das organizações e de valores humanos. O ensino deve corresponder à visão transformativa atual onde os "designers lideram em vez de seguir" (Fry, 2013), ou seja, designers devem ser profissionais redirecionadores, que desafiam e até subvertem o status quo, podendo criar mudanças significativas por meio de ações projetuais que catalisem as transformações necessárias, contribuindo para estabelecer um futuro viável (Franzato, 2016; Guellerin, 2009; Bonsiepe, 2013). Envolver outras pessoas, práticas, valores e futuros demanda diferentes fundamentos e esta construção é responsabilidade da educação e pesquisa de design.

Sob o ponto de vista de Ehn (2018), o designer deve atuar como um ativista para desenhar e controlar a tecnologia. Ele aponta a "intra-ação" com os atores envolvidos no projeto como a competência mais importante para os designers, pois não se espera que haja um consenso de ideias ao trabalhar de maneira coletiva com pessoas que tem interesses diversos. Quanto ao ensino, Ehn sugere que, o designer deve aprende a atuar em condições de incerteza, explorar o desconhecido, onde o desafio é adquirir a habilidade para navegar e seguir avançando. Nesta abordagem a aceitação do erro e dos imprevistos é inerente a esta maneira de agir sendo que estas habilidades não são encontradas nos métodos objetivos, e sim num repertório de práticas situadas.

Nesta abordagem a autonomia também se torna uma característica fundamental, pois a atuação do design em nível sistêmico requer a busca e organização de fontes de informação e conhecimento, e a capacidade de tomar decisões compreendendo a responsabilidade sobre suas escolhas, como forma de intervir no mundo.

\section{Discussão}

A elaboração de programas de design se torna uma tarefa difícil para as escolas de design que pretendem uma abordagem atual reconhecendo a complexidade e variedade de opções possíveis para a atuação do design. Para Imbesi (2011), esta é uma oportunidade de elaborar programas de design para produzir, reunir e dar visibilidade a experiências de design crítico, emergindo como um espaço alternativo e espontâneo, muitas vezes paralelamente e em correlação com a produção oficial estabelecida.

A nova escola tende a ser múltipla como múltiplo é o modelo de globalização; deve ser transversal e "atravessável"; deve fornecer conteúdos culturais, históricos, críticos e reflexivos em maior escala, incorporar valores mais humanistas que tecnicistas e com mais conteúdos experimentais que previsíveis (Celaschi e Moraes, 2013).

Portanto, os educadores de design tendem a colocar mais ênfase no processo de aprendizagem contínua de novas habilidades e conhecimentos, reorientando o papel do design e da educação em design no processo de desenvolvimento do projeto (Liem e Sigurjonsson, 2014). Nestas circunstâncias, o objetivo de educar estudantes de design nas universidades tem sido o 
cultivo de habilidades em gestão do design, resolução de problemas, aprendizagem ao longo da vida e o pensamento e a prática reflexiva (Schön, 1988) para que possam adaptar-se melhor às futuras mudanças e desafios. Isto não só implica que a pesquisa acadêmica leve a novos conhecimentos, mas também que os próprios produtos e artefatos concebidos devem ser considerados uma forma de conhecimento (Frayling, 1993).

Estas mudanças colocam o design em constante estado de experimentação, onde as mudanças são sempre contínuas e não se pode enquadrar um problema como certo e uma solução como pronta. Nesta abordagem as novas competências compostas por conhecimentos, habilidades e atitudes, também sofrerão mudanças contínuas, assim como o ensino de design. Portanto justifica-se a elaboração de competências preliminares (fig. 2) que estão vinculadas a um enquadramento momentâneo das possíveis praticas.

Figura 2: novas práticas e competências para o design pós-industrial

\begin{tabular}{|l|l|}
\hline Práticas do design pós-industrial & Novas competências para o design \\
\hline Produção e design distribuídos & $\begin{array}{l}\text { Colaboração, visão crítica, identificação de } \\
\text { oportunidades, pensamento em rede }\end{array}$ \\
\hline Complexidade & $\begin{array}{l}\text { Identificação de problemas complexos, } \\
\text { busca de conexões em outras áreas }\end{array}$ \\
\hline Fronteiras difusas & $\begin{array}{l}\text { Transito transdisciplinar, aprendizagem ativa } \\
\text { e abertura para experimentação }\end{array}$ \\
\hline Novos híbridos de design & $\begin{array}{l}\text { Co-criação de cenário futuro, gestão, } \\
\text { negociação }\end{array}$ \\
\hline Inovação em modelos de negócio & $\begin{array}{l}\text { Agenciamento de mudança, pensamento } \\
\text { holístico, articulador de inovação social }\end{array}$ \\
\hline Novas formas de atribuir valor & $\begin{array}{l}\text { Mediação, facilitação, visualização de } \\
\text { novas oportunidades }\end{array}$ \\
\hline Transformação de contextos & $\begin{array}{l}\text { Empreendedorismo com capacidade de } \\
\text { trabalhar em rede }\end{array}$ \\
\hline Conexão entre espaços virtuais e físicos & $\begin{array}{l}\text { Autonomia, facilitação de processos e mediação } \\
\text { de relações }\end{array}$ \\
\hline Auto-produção & $\begin{array}{l}\text { Elaboração, coleta e analise de dados; } \\
\text { personalização de produtos }\end{array}$ \\
\hline Design em rede & Indústria 4.0
\end{tabular}

Fonte: A autora

Para Brown \& Adler (2008) torna-se necessário construir práticas distribuídas e reflexivas nas quais as experiências sejam coletadas, examinadas, agrupadas, comentadas e testadas em novos contextos. Podemos chamar isso de "aprender sobre o aprendizado", uma operação de arranque na qual os educadores, juntamente com os alunos, estão aprendendo entre eles. Isso pode se tornar uma infraestrutura viva e dinâmica - um exercício prático reflexivo (Brown \& Adler, 2008).

Em suma pode-se concluir que não existe o processo formativo ideal, mas existe um caminho por meio do qual a realidade é continuamente restabelecida para experimentar ininterruptamente a integração entre novos "saberes", novos "saber fazer" e novos "saber ser", assim como novos entrelaçamentos entendidos como estimulação contínua da identidade do designer e dispositivos para a liberação de sua criatividade expressiva (Celaschi e Moraes, 2013). 


\section{Conclusão}

Se ensinar exige uma reflexão crítica sobre a prática e a compreensão de que a educação é uma forma de intervenção no mundo, conclui-se que existe uma necessidade premente em repensar o ensino de design. Na busca por um design transformador que corresponda as novas perspectivas e necessidades da sociedade contemporânea, torna-se necessário a reflexão sobre quais conhecimentos, habilidades e atitudes necessários aos novos designers. Este ensino deve promover a compreensão e consciência do contexto global onde os alunos irão trabalhar permitindo que estes possam refletir sobre a evolução dos sistemas e se responsabilizar sobre sua futura interferência no sistema sócio técnico. A mudança do ensino do design por meio de uma abordagem sistêmica, colaborativa, e proativa, deve modificar as práticas disciplinares, instigando e valorizando as práticas críticas e reflexivas.

O ensino deve ser formativo e transformador, flexível e ágil, possibilitando sua rápida renovação e reconfiguração para acompanhar as mudanças tecnológicas, sociais e econômicas. Conclui-se também que o ensino deve possibilitar e providenciar espaço para práticas experimentais, propiciando o design de novos contextos e sistema de produção baseado numa redefinição de papéis entre produtor, consumidor e designer.

Partindo deste pressuposto, as tecnologias digitais integradas oferecem oportunidades e desafios para o ensino. Se inseridas ao ensino apenas como habilidade técnica e ferramenta de projeto, continuaremos a formar designers industriais vinculados aos paradigmas da sociedade industrial, limitando a compreensão dos estudantes sobre seu papel como cocriadores do futuro e os distanciando da dinâmica de transformações sociais, culturais, econômicas e tecnológicas. Para formar profissionais críticos, reflexivos, com visão estratégica, questionadores do porquê, o que e como produzir, capazes de elaborar novos modelos de negócio e lógicas de valor.

Transformar requer uma visão holística do sistema atual para analisar as possibilidades, avaliar os caminhos e escolher uma forma de abordar o que se apresenta responsabilizando-se pelo resultado das escolhas feitas durante o processo. O designer não deve ser apenas o solucionador de problemas, aquele que escolhe a melhor solução possível, deve sim ter um papel transformador, propondo inovações disruptivas que possam mudar o sistema que anteriormente o mantinha refém das tecnologias e dinâmicas sociais e econômicas de produção e consumo.

O ensino deve oferecer novas práticas pedagógicas, novas competências e introduzi-las na matriz curricular para que atendam à atual complexidade que se apresenta para o design. Mas além destas é preciso refletir sobre cenários futuros para ampliar a visão sobre possíveis tendências que afetarão a sociedade nos próximos anos e trabalhar com estas temáticas no desenvolvimento de projetos de design de transformação.

\section{Referências}

ARQUILLA V.; BIANCHINI M.; MAFFEI S. Designer=Enterprise. A new policy for the next generation of Italian designers, Proceedings International Design Management Symposium, Tsinghua Hong Kong, DMI, 2011. (https://www.academia.edu/7549731/Designer_Enterprise_A_new_policy_for_ the_next_generation_of_Italian_designers) 
BIANCHINI, Massimo. Industrious Design. The role of design in the evolution of (micro) production models enabled by the hybridization of individuals and organizations. Tese de doutorado em design. Politécnico de Milão, 2014 p. 337

BONSIEPE, Gui. Design cultura e sociedade. São Paulo: Blucher, Brasil, 2013.

BORJA DE MOZOTA, B. Gestão do Design. Porto Alegre: Bookman, 2011.

BUCHANAN. Design Research and the New Learning. Design Issues, 17(4), pp. 3-23, 2001.

BROWN, John S; ADLER, Richard P. Minds on Fire: Open Education, the Long Tail, and Learning 2.0. Educause rewiew, vol. 43, no 1, 16-32, 2008. (http://er.educause.edu/articles/2008/1/minds-onfire-open-education-the-long-tail-and-learning-20)

BRUNS, Axel. Produsage: Towards a Broader Framework for User-Led Content Creation. Proceedings Creativity \& Cognition 6, Washington, DC, 2007 (https://eprints.qut.edu.au/ 6623/1/6623.pdf)

CELASCHI, Flaviano; FORMIA, Elena. Education for design processes: the influence of Latin cultures and contemporary problems in production systems. Innovation in design education: Theory, research and processes to and from a Latin perspective. Torino, 2012

CELASCHI, Flaviano; MORAES, Dijon de. Futuro, Bem-Estar, Interdependência: Palavras-Chave para o Design Contemporâneo. In: MORAES, Dijon de; CELASCHI, Flaviano (Org.). Caderno de Estudos Avançados em Design: Design e Humanismo. v. 7. Barbacena: EdUEMG, 2013. p. 35-60.

CELASCHI, Flaviano. Advanced design driven approaches for $\mathbf{4 . 0}$ Industry framework. Human centered dimension in fourth digital-industrial revolution, Strategic Design Research Journal, v.10, n. 2, pp. 97-104, 2017. (http://revistas.unisinos.br/index.php/sdrj/article/view/sdrj.2017)

DE VERE, lan. Industrial Design 2.0: A Renaissance. International conference on engineering and product design education 5 \& 6, Dublin Institute of Technology, Dublin, 2013.

DIAS, Pedro J.J.S. Design e autoprodução. Novos paradigmas para o design de artefatos na sociedade pós-industrial. A contribuição das tecnologias digitais. Doutoramento em Belas Artes. Especialidade de Design de Equipamentos. Universidade de Lisboa, 2014

DUBBERLY, Hugh. A proposal for the future of design education. Design Education Manifesto, ICOGRADA, 2011 (http://www.dubberly.com/articles/design-education-manifesto.html)

EHN, P.; FARÍAS, I.; SÁNCHEZ Criado, T. La posibilidad de que cosas del diseño puedan ser socialistas-democráticas: Entrevista a Pelle Ehn. Entrevistadores: I. Farías \& T. Sánchez Criado. Diseña, (12), 52-69, 2018

FINDELI, Alain. Rethinking design education for the 21st century: Theoretical, methodological and ethical discussion. Design Issues, v. 17, n. 1, p. 5-17, 2001.

FRANZATO, C. Articulação em rede para "um mundo melhor". Entrevista especial com Carlos Franzato por: Márcia Junges. Revista IHU On-Line, no. 493, 2016. (http://www.ihu.unisinos.br/159noticias/entrevistas/560190-articulacao-em-rede-para-um-mundo-melhor-entrevista-especialcom-carlo-franzato)

FRAYLING, Christopher. Research in Art and Design. Research Papers: Vol 2. London, UK: Royal College of Art, 1993. 
FRY, Tony. A Decade of Design Education, Australian Design Review.Tom Inns, ed., Introduction. Designing for the 21st Century, vol. 2, Interdisciplinary Methods and Findings, Ashgate, 2010.

GUELLERIN, Christian. Design education and globalisation; the new deal, 2009. (http://blogs.lecolededesign.com/christianguellerin/2009/05/14/design-education-andglobalisation-the-new-deal-part-iv-tremendous-evolution-of-training-courses-in-design-workbased-education-brought-to-the-fore/?lang=en)

HERMANN, Mario; PENTEK, Tobias; OTTO, Boris. Design principles for industrie 4.0 scenarios: a literature review. Working Paper n. 01/2015. Technische Universität Dortmund. Fakultät Maschinenbau. Audi Stiftungslehrstuhl Supply Net Order Management, 2015.

IMBESI, Lorenzo. Design for post-industrial societies: re-thinking research and education for contemporary innovation. Ottawa, Carleton University, SID - School of Industrial Design, 2011

KAUFMAN, J. Why design education? Infrastructure issues affecting the future of industrial design education. IDSA National Education Conference ,1998.

KRUCKEN, L. Design e território: valorização de identidades e produtos locais. São Paulo: Studio Nobel, 2009

LIEM, A.; SIGURJÓNSSON, J. B. Positioning Industrial Design Education within Higher Education: How to face increasingly challenging market forces? Uniped ârgang 37, side 44_572, 2014.

LOY, Jennifer. The future for design education: preparing the design workforce for additive manufacturing. International Rapid Manufacturing, V.5, n. 2, 2015

MANZINI, Ezio. Design Schools as Agents of (Sustainable) Change: A Design Labs Network for an Open Design Program. In: INTERNATIONAL SYMPOSIUM FOR DESIGN EDUCATION RESEARCHERS, 1, 2011, Paris, França. Researching Design Education: Symposium Proceedings. Aalto, Finland: Cumulus Association/Design Research Society - DRS, p. 9-16, 2011.

MENICHINELLI, Massimo. A Framework for Understanding the Possible Intersections of Design with Open, P2P, Diffuse, Distributed and Decentralized Systems. Disegno Journal of Design Culture, 2016. (http://disegno.mome.hu/articles/2016/Disegno2016_1_2_04_Menichinelli.pdf)

MINEIRO, Érico F. Experimentação em design como estratégia no cenário da autoprodução. Tese de doutorado. 201p - Pontifícia Universidade Católica do Rio de Janeiro, Departamento de Artes e Design, 2016

NICOLESCU, Basarab. A evolução transdisciplinar a Universidade condição para o desenvolvimento sustentável. Conferência no Congresso International "A Responsabilidade da Universidade para com a Sociedade", International Association of Universities, Chulalongkorn University, Bangkok, Thailand, 1997 (http://ciret-transdisciplinarity.org/bulletin/b12c8por.php)

OECD, The Next Production Revolution: Implications for Governments and Business, OECD Publishing, Paris, 2017. (http://www.keepeek.com/Digital-Asset-Management/oecd/science-andtechnology/the-next-production-revolution_9789264271036-en\#page4)

POGGENPOHL, Sharon H. Envisioning a Future Design Education: an Introduction. Visible Language, v. 46, n. 1/2, p. 8-18, 2012.

SCHÖN, D. A. Designing: Rules, types and worlds. Design Studies, 9(3), 181-190, 1988 
SCHUH, G; RUDOLF, S; RIESENER, M. Design for Industrie 4.0. International Design Conference Design 2016. Dubrovnik - Croatia, May 16 - 19, 2016.

TROXLER, Peter. Beyond Consenting Nerds. Lateral Design Patterns for New Manufacturing. Hogeschool Rotterdam Uitgeverij, 2015. (http://www.petertroxler.net/wp-content/uploads /2015/11/Internetversie-Peter-Troxler.pdf)

WILSON, Stephanie; ZAMBERLAN, Lisa. Design for an unknown future. Amplified roles for collaboration. New Design Knowledge and Criativity. Design Issues. v. 31, n2, p3-15, 2015.

WHITELEY, N., O Designer Valorizado, Arcos, 1, único, pp. 73-95, 1998

WOOD, John. The shortening of design futures. An interpretation of Aristotles's belief in design as a 'final cause', 2012 (http://metadesigners.org/Shortening-of-Design-Futures) 